\title{
Poincaré's Observation and the Origin of Tsallis Generalized Canonical Distributions
}

\author{
C. Vignat* and A. Plastino** \\ *L.I.S. Grenoble, BP 46, 38402 St. Martin d'Hères cedex, France, vignat@lis.inpg.fr and \\ **Facultad de Ciencias Exactas, National University La Plata \\ and IFLP-CONICET, C. C. 727, 1900 La Plata, Argentina
}

\begin{abstract}
In this paper, we present some geometric properties of the maximum entropy (MaxEnt) Tsallisdistributions under energy constraint. In the case $q>1$, these distributions are proved to be marginals of uniform distributions on the sphere; in the case $q<1$, they can be constructed as conditional distributions of a Cauchy law built from the same uniform distribution on the sphere using a gnomonic projection. As such, these distributions reveal the relevance of using Tsallis distributions in the microcanonical setup: an example of such application is given in the case of the ideal gas.
\end{abstract}

PACS numbers: 05.40.-a, 05.20.Gg 


\section{INTRODUCTION}

Nonextensive thermostatistics is a very active field nowadays, based on the concept of Tsallis' information measure (or entropy) [1]. The concomitant maximum (Tsallis) entropy distributions play a role for the description of non-extensive systems akin of that of Gaussian ones for extensive systems. By Tsallis distributions, we mean the following $n$-variate probability densities:

- if $\frac{n}{n+2}<q<1$, with $\beta=\frac{1}{2 q-n(1-q)}$ and $A_{q}=\frac{\Gamma\left(\frac{1}{1-q}\right)(\beta(1-q))^{n / 2}}{\Gamma\left(\frac{1}{1-q}-\frac{n}{2}\right) \pi^{n / 2}|C|^{1 / 2}}$

$$
f_{X}(X)=A_{q}\left(1-\beta(q-1) x^{T} C^{-1} x\right)^{\frac{1}{q-1}}
$$

- if $q>1$ or $q<0$, with $\beta=\frac{1}{2 q-n(1-q)}$ and $A_{q}=\frac{\Gamma\left(\frac{q}{q-1}+\frac{n}{2}\right)(\beta(q-1))^{n / 2}}{\Gamma\left(\frac{q}{q-1}\right) \pi^{n / 2}|C|^{1 / 2}}$

$$
f_{X}(X)=A_{q}\left(1-\beta(q-1) x^{T} C^{-1} x\right)_{+}^{\frac{1}{q-1}}
$$

where $x_{+}$denotes $\max (x, 0)$.

These distributions are solutions of the following maximization problem:

$$
\max _{f} \frac{1}{1-q} \int_{\mathbb{R}^{n}} f^{q} \text { such that } \int_{\mathbb{R}^{n}} x x^{T} f(x) d x=C .
$$

In this sense, they are the counterparts of the Gaussian distribution with covariance matrix $C$ which is solution of

$$
\max _{f}\left(-\int_{\mathbb{R}^{n}} f \log f\right) \text { such that } \int_{\mathbb{R}^{n}} x x^{T} f(x) d x=C
$$

Gaussian distributions exhibit universal properties that allow to derive important statistical results such as the central limit theorem or the entropy power inequality. Some of these results have a "Tsallis-counterpart" [1]. A geometric approach like the one advanced here is interesting since it sheds new light on these properties.

\section{GEOMETRIC APPROACH}

\section{A. stochastic properties}

In this section, we propose a geometric characterization of Tsallis distributions based on their stochastic properties. Thus we begin by a detailed stochastic study of Tsallis random vectors. We denote as

$$
X \sim T_{q, C}
$$

a Tsallis vector with nonextensivity parameter $q$ and covariance matrix $C$. 
case $\frac{n}{n+2}<q<1$

If $X \sim T_{q, C}$, then a stochastic representation of $X$ writes

$$
X=\sqrt{m-2} C^{1 / 2} \frac{N}{\sqrt{a}}
$$

where $a$ is a chi-square distributed random variable with $m=\frac{2}{1-q}-n$ degrees of freedom, independent of the Gaussian random vector $N$ with unit covariance matrix. It is understood that $m$ may be non-integer.

duality result

If $X \sim T_{q, C}$ with $\frac{n}{n+2}<q<1$ then the following random vector

$$
Y=\frac{X}{\sqrt{1+\frac{1}{m-2} X^{T} C^{-1} X}}
$$

verifies

$$
Y \sim T_{q *, C *}
$$

with

$$
C *=C \frac{m-2}{m+n}, \frac{1}{q *-1}=\frac{1}{1-q}-\frac{n}{2}-1
$$

case $q>1$

From the duality result, we deduce a stochastic representation for $Y \sim T_{q *, C}$ with $q *>1$ as follows

$$
Y=\sqrt{m+n} C^{1 / 2} \frac{N}{\sqrt{a+N^{T} N}}
$$

where $a$ is a chi-square random variable with $m=\frac{2 q *}{q *-1}$ degrees of freedom, independent of the Gaussian random vector $N$ with unit covariance matrix.

\section{B. Geometric representation}

The preceding stochastic representations allow to derive easily a geometric construction of Tsallis random vectors as follows. 
case $q>1$

An $n$-variate random vector $U$ is uniformly distributed on the ellipsoid $\mathcal{E}_{C, n}=$ $\left\{Z \in \mathbb{R}^{n} \mid Z^{T} C Z=1\right\}$ if and only if it writes

$$
U=\frac{C^{-1 / 2} N}{\sqrt{N^{T} N}}
$$

where $N$ is a Gaussian vector with unit covariance matrix. Comparing this stochastic representation with the Tsallis vector's one (11), the following result can be proved:

Theorem 1 If $Y \sim T_{q *, C}$ with $q *>1$ or $q *<0$ then $Y$ is the $n$-variate marginal vector of an $(m+n)$-variate random vector $U$ uniformly distributed on the ellipsoid $\mathcal{E}_{C^{-1}, m+n}$ with $m=\frac{2 q *}{q *-1}$.

We remark that, according to this characterization, the $(m+n-1)$-variate marginal random vector is the only one distributed according to a Tsallis law with negative non-extensivity index (namely $q *=-1)$; moreover, the $(m+n-2)$-variate marginal is uniform inside a $(m+n-2)$ dimensional ellipsoid and corresponds to a non-extensivity index $q *=+\infty$. All other lower dimension marginals have finite and positive index $q *>1$.

case $q<1$

For the sake of simplicity, we address here the uncorrelated case $C=I_{n}$. We note that this is not a loss of generality, since

$$
X \sim T_{q, C} \Rightarrow C^{-1 / 2} X \sim T_{q, I}
$$

Theorem 2 Assume point $P$ is uniformly distributed on the sphere $\mathcal{E}_{I_{n}, n}$; consider the intersection $M$ of vector $O P$ with the hyperplane $\mathcal{H}=\left\{Z \in \mathbb{R}^{n} \mid Z_{n}=1\right\} .{ }^{1}$ Then point $M$ follows $a(n-1)-$ variate Cauchy distribution in $\mathcal{H}$ :

$$
f_{M}\left(y_{1}, \ldots, y_{n-1}\right) \propto\left(1+\sum_{i=1}^{n-1} y_{i}^{2}\right)^{-\frac{n}{2}}
$$

Moreover, any distribution of point $M$ conditioned on variables $y_{k+1}, \ldots, y_{n-1}$ follows a $k-$ variate Tsallis distribution with $m=n-k$ degrees of freedom [10]:

$$
f_{M}\left(y_{1}, \ldots, y_{k} \mid y_{k+1}, \ldots y_{n-1}\right) \propto\left(1+\lambda \sum_{i=1}^{k} y_{i}^{2}\right)^{-\frac{n}{2}}
$$

\footnotetext{
${ }^{1}$ point $\mathrm{M}$ is called the gnomonic projection of point $\mathrm{P}$
} 


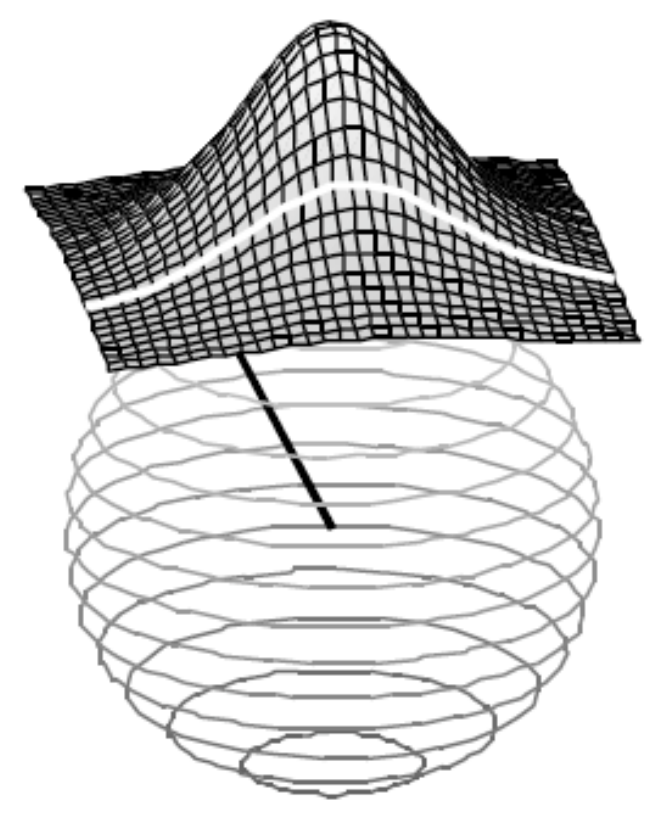

FIG. 1: the $n=3$ dimensional sphere, the Cauchy distribution and one of its conditional laws, the Tsallis $q=\frac{1}{3}$ distribution

In Fig. 1 below, the $n=3$ dimensional sphere is depicted, with the Cauchy distribution of point $M$ : the white curve represents, up to a constant, the one dimensional conditional density $f_{M}\left(y_{1} \mid y_{2}\right)$ which is Tsallis distributed with $m=2$ degrees of freedom $\left(q=\frac{1}{3}\right)$.

\section{COMMENTS ON SOME PARTICULAR CASES}

In this section, we comment on the geometric results obtained.

\section{A. the case $q>1$ and Poincaré's observation}

The characterization of Tsallis random vectors - with $q>1$ and identity covariance matrix as marginals of vectors uniformly distributed on a sphere can be related to a result attributed to Poincaré called "Poincaré's Observation" [2] (for a discussion on this paternity, see [8]).

Theorem 3 If $X=\left(x_{1}, \ldots, x_{p}\right)$ is uniformly distributed on the sphere $S_{p}$ with radius $\sqrt{p}$, then 
for fixed $n<+\infty$,

$$
\lim _{p \rightarrow \infty} \operatorname{Pr}\left(\cap_{i=1}^{n}\left(a_{i} \leq x_{i} \leq b_{i}\right)\right)=\int_{a_{1}}^{b_{1}} \frac{e^{-\frac{x^{2}}{2}}}{\sqrt{2 \pi}} d x \ldots \int_{a_{n}}^{b_{n}} \frac{e^{-\frac{x^{2}}{2}}}{\sqrt{2 \pi}} d x
$$

This result shows that a Gaussian (Tsallis $q=1$ maximizer) random vector with fixed dimension $n$ can be considered as the marginal vector of an infinite-dimensional vector uniformly distributed on the sphere $S_{\infty}$. The results obtained above demonstrate that, in contrast, a Tsallis $q>1$ random vector of dimension $n$ can be viewed as the marginal of a finite-dimensional vector uniformly distributed on the sphere $S_{p}$ with

$$
p=\frac{2 q}{q-1}+n
$$

As $q \rightarrow 1^{+}, p \rightarrow+\infty$ and we recover Poincaré's observation.

\section{B. the case $m=1, q<1$}

The case $m=1$ and $q<1$ corresponds to Cauchy-Lorentz distributions, which are not stricto sensu Tsallis distributions since they have no finite covariance matrix. However, they appear in many relevant physical situations [4]. They are defined as

$$
f_{X}(X)=\frac{\Gamma\left(\frac{n+1}{2}\right)}{\pi^{\frac{n+1}{2}}}\left(1+X^{T} X\right)^{-\frac{n+1}{2}}
$$

and have for stochastic representation

$$
X=\frac{N}{M}
$$

where $N$ is an $n$-variate Gaussian vector and $M$ is a scalar Gaussian variable, independent of $N$. Applying the above results, we deduce that they can be obtained as gnomonic projections of uniformly distributed points on the $n$-sphere, as illustrated in the following figure in the case $n=3$ : as point $P$ describes uniformly the surface of $S_{3}$, point $M_{2}$ in the plane $X_{3}=1$ is distributed with Tsallis law $T_{1 / 3, I_{2}}$.

\section{APPLICATION: THE IDEAL GAS}

We adopt here notations of Minlos [5]. Denote as $q_{i}$ the velocity of the i-th particle among $N$ of an ideal gas in volume $\Lambda$. Let us define the set

$$
\Omega_{\Lambda, N, E}=\left\{\left(q_{1}, \ldots, q_{N}\right) \mid H\left(q_{1}, \ldots, q_{N}\right)=E\right\}
$$




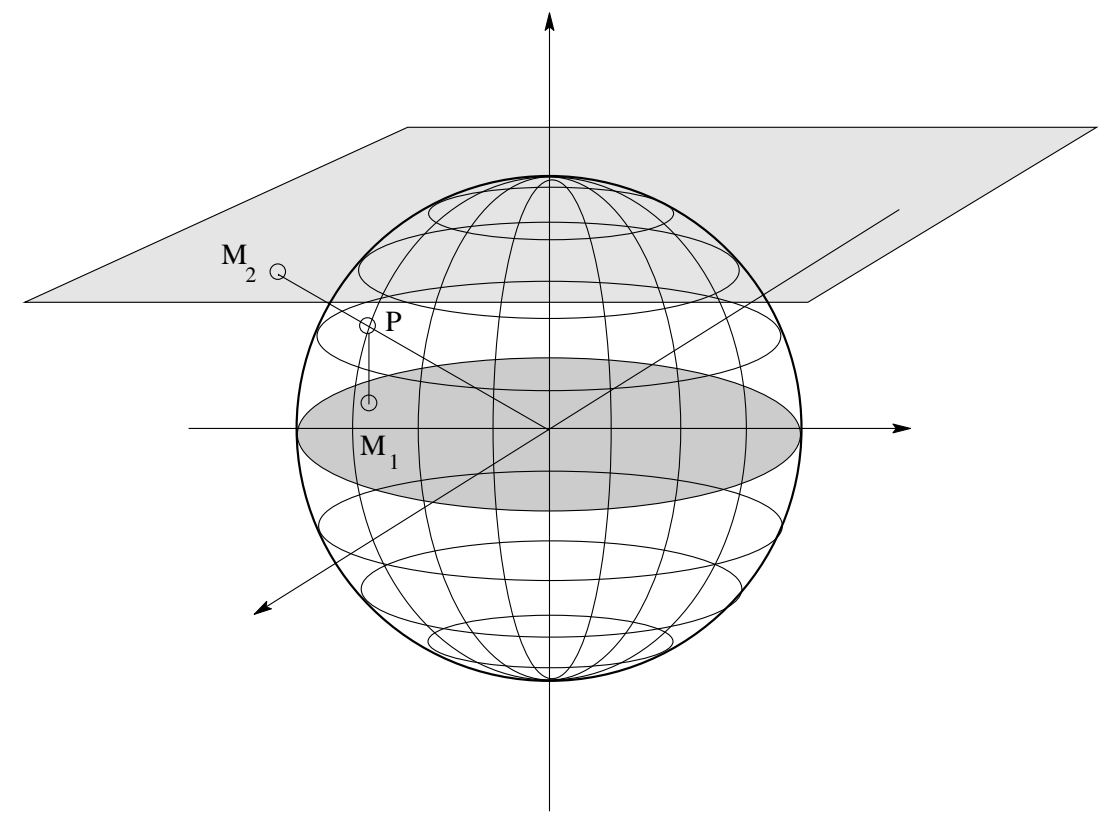

FIG. 2: point $M_{2}$ distributed with Tsallis law $T_{1 / 3, I_{2}}$ while point $M_{1}$ is distributed with Tsallis law $T_{-1, I_{2}}$ where the Hamiltonian writes

$$
H\left(q_{1}, \ldots, q_{N}\right)=\sum_{i=1}^{N} \frac{1}{2} m q_{i}^{2} .
$$

In the microcanonical setup, one assumes that the velocities have a distribution $\nu$ uniform on surface $\Omega_{\Lambda, N, E}$ :

$$
\nu\left(q_{1}, \ldots, q_{N}\right)=\frac{2|\Lambda|^{N} \pi^{3 N / 2}}{m \Gamma\left(\frac{3 N}{2}\right)}\left(\frac{2 E}{N}\right)^{\frac{3 N}{2}-1} .
$$

Let us now consider a subsystem of the gas, consisting of $N_{0}<N$ particles in a volume $\Lambda_{0} \subset \Lambda$ : their distribution can be easily computed as

$$
\nu\left(q_{1}, \ldots, q_{N_{0}}\right) \propto\left(E-\sum_{i=1}^{N_{0}} \frac{1}{2} m q_{i}^{2}\right)_{+}^{\frac{3}{2}\left(N-N_{0}\right)-1} .
$$

A usual approach consists then in taking the thermodynamic limit: assuming the total volume $\Lambda$ converges to $\mathbb{R}^{3}$, the number of particle per volume $\frac{N}{|\Lambda|}$ converges to density $\rho$ and the energy per volume $\frac{E}{|\Lambda|}$ converges to energy density $e$, we deduce

$$
\nu\left(q_{1}, \ldots, q_{N_{0}}\right) \propto \exp \left(-\frac{3 \rho}{2 e} \sum_{i=1}^{N_{0}} \frac{1}{2} m q_{i}^{2}\right)
$$

and recover the celebrated Boltzmann distribution of velocities. 
If, however, we do not take the thermodynamic limit but remain under the finite-dimensional assumption, then we remark that distribution (2) is an $N_{0}$-variate Tsallis distribution with nonextensivity index

$$
q=\frac{N-N_{0}}{N-N_{0}-\frac{2}{3}}>1
$$

In other terms, the distribution of the finite dimensional ideal gas in the microcanonical ensemble maximizes Tsallis entropy with parameter $q$ as defined by (3). As $N-N_{0}$ is exactly the dimension of the heat bath seen by the $N_{0}$ particles, we may conclude that, in this example, Tsallis entropy appears as the finite-dimensional counterpart of Shannon entropy. This idea was already derived by one of the authors [6] years ago, but without the details we provide here. Moreover, Adib et al. [7] have characterized Tsallis $q>1$ distributions as marginals of uniform distributions, in the case of more general Hamiltonians verifying an homogeneity property, but they assume the (stronger) ergodic hypothesis to derive this result.

[1] M. Gell-Mann and C. Tsallis, Eds. Nonextensive Entropy: Interdisciplinary applications (Oxford University Press, Oxford, 2004); A.R. Plastino and A. Plastino, Phys. Lett. A 177 (1993) 177.

[2] H. Poincaré, Calcul des Probabilités, Gauthier-Villars, 1912

[3] H.P. McKean, Geometry of Differential Space, The Annals of Probability, 1973, vol.1, n.2, pp.197-206

[4] S. Abe, A.K. Rajagopal, Information theoretic approach to statistical properties of multivariate Cauchy-Lorentz distributions, J. Phys. A: Math. Gen. 34 (2001)

[5] R.A. Minlos, Introduction to mathematical statistical physics, Providence, R.I. : American Mathematical Society, (2000)

[6] A.R. Plastino, A. Plastino, Phys. Lett. A, 193 (1994), 251

[7] A.B. Adib, A.A. Moreira, J.S. Andrade Jr., M.P. Almeida, Tsallis thermostatistics for finite systems: a Hamiltonian approach, Physica A 322 (2003) 276- 284

[8] P. Diaconis, D. Freedman, A dozen de Finetti-style results in search of a theory, Ann. Inst. H. Poincaré Probab. Statist. 23 n.2, suppl. 397-423 (1987)

[9] D. Song, A. K. Gupta, Lp-norm uniform distribution, proceedings of the american mathematical society, Vol. 125, Number 2, February 1997, Pages 595-601

[10] M. E. Johnson, Multivariate Statistical Simulation, Wiley and Sons, (1987) 